\title{
Self-organized control of an tendon driven arm by differential extrinsic plasticity
}

\author{
Georg Martius ${ }^{1}$, Rafael Hostettler ${ }^{2}$, Alois Knoll ${ }^{2}$, and Ralf Der $^{3}$ \\ ${ }^{1}$ IST Austria, Am Campus 1, 3400 Klosterneuburg, Austria \\ ${ }^{2}$ Institut für Informatik VI, TU München, Boltzmannstr. 3, 85748 Garching bei München, Germany \\ ${ }^{3}$ Max Planck Institute for Mathematics in the Science, Inselstr. 22, 04103 Leipzig, Germany \\ gmartius@ist.ac.at
}

The self-determined cognitive development of highcomplexity autonomous robots is a challenging task for both the creation of robot-human ecosystems and the creation of artificial life systems with real, human-like robots. Anthropomimetic robots are a prominent example of this challenge. Different from classical robots, anthropomimetic robots are built following the morphology of the human body. Such robots are more soft than classical systems making them safer to interact with and thus favorable for service robots in human environments. Moreover, because of their human like morphology, they can be used for better understanding human behavior generation and development.

World wide, several of such muscle-tendon driven (MTD) systems have already been built. While mechatronically at an advanced level, the control of both MTD and soft robotic systems in general is still in its infancy. A generic example is given by pertinent $\mathrm{EU}$ projects ranging from CRONOS, to ECCEROBOT to MYOROBOTICS. While excellent work has been done in building these robots their control faces many problems. Learning of control policies becomes essential and is investigated mainly in the reinforcement learning setting.

Without a very compact parametrization learning a new behavior takes a very long time in high-dimensional systems. This situation clearly calls for new controller paradigms which optimally exploit the physical properties of such soft systems as indicated by embodied AI. This paper presents an approach that includes the world-i. e. body plus environment-more actively and more systematically in the control process than other embodied control approaches. By inverting the roles of the controller and the controlled, the world becomes not only "its own best model" (Rodney Brook's idea) but is leveraged to "its own best controller" (Der and Martius, 2016). This idea can be implemented by a neural network with a novel synaptic plasticity rule (Der and Martius, 2015, 2016), as shown in Fig. 1.

The novelty of the controller can be demonstrated best by applying it to MTD systems, for instance the Myo-robotics arm, reported here, with its ball and socket shoulder joint and 9 muscles in total. Different from classical robots with

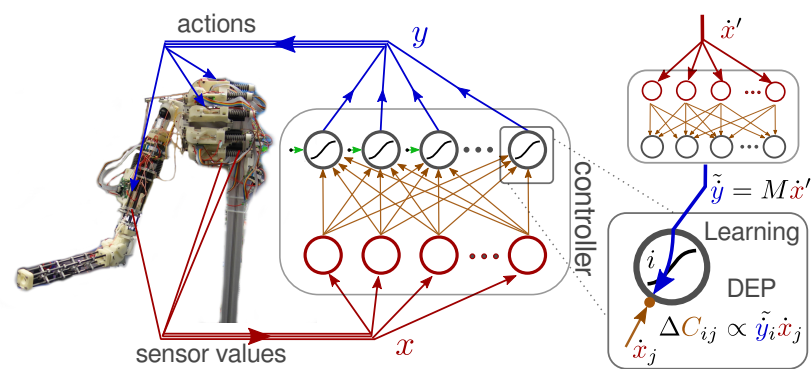

Figure 1: Neural controller network connected to the Myo-robotic arm. The inset on the right illustrates the synaptic plasticity, called differential extrinsic plasticity, which is driven by a modified differential Hebbian law, multiplying the time derivatives of the incoming sensor values $\dot{x}$ with the virtual motor values $\tilde{\dot{y}}$, which are generated by the inverse model ( $M$, one-to-one mapping in the case of the arm) from next input's derivative $\dot{x}^{\prime}$.

revolute joints, the motor positions do not directly translate into joint angles and into poses. Due to the elasticity of the muscles, there are infinitely many combinations of motor positions for a single arm pose. Apart from that, the most challenging task is to avoid the dislocation of the shoulder which cannot happen with revolute joints. Astonishingly, although structurally extremely simple, the new control paradigm does not have problems with these particularities. For instance the tendons are kept tight automatically, such that no dislocation appears. When embedding our controller, see Fig. 1] into the sensorimotor loop, a meta-system-consisting of the mechanical system, the controller with its sensor driven synaptic dynamics, and the energy supply (battery) — is created displaying a rich behavioral spectrum like limit cycle attractors, long lived transients, and fixed point flows generating pseudo-random sequences of poses. The concrete behavior is not given explicitly, but specific behaviors develop by themselves in a dynamical interplay between controller dynamics and world dynamics. This open physical system is like a reservoir of meta-stable behavior patterns waiting to be excited. Ex- 
citation can be achieved either by manual interaction (see below) or by the self-amplification of latent modes spontaneously arising in physical subsystems, see Video 1 (see playfulmachines.com/MyoArm-2). By way of example, consider Video 3 where a weight (a bottle) was suspended from the tip of the arm with a string, forming as a physical subsystem. In the beginning, minimal motor activities are seen to spontaneously excite minor pendulum motions. These oscillations directly exert physical forces on the arm which propagate via the springs into the sensor values and eventually into the synaptic dynamics which governs the behavior. This may lead to the amplification of latent pendulum modes until a stable circular movement of the pendulum is achieved. These findings elucidate how a physical subsystem (the pendulum) may pilot—by its internal dynamicsthe meta-system into a resonant state, i. e. a whole-system mode with defined frequency.

Actually, this is the essence of the method which explains the emergence of specific modes-specific for the actual physical setting - of the system. For instance, when attaching a bottle half-filled with water to the tip of the arm in either horizontal or vertical orientation, stable shaking modes are arising, as demonstrated by both Video 6 and Video 7 showing modes specific for each physical setting (horizontal or vertical bottle). Again, we see how the meta-system may become resonant with the internal dynamics of a subsystem, if the latter provides perceivable correlations over space and time. This is the case for instance when the water is hitting either the walls or top and bottom of the bottle. These impacts cause a reaction of the springs and hence of the sensor values, which may increase correlations in the synaptic dynamics resulting in enhances motions of the arm in coherence with these signals.

By this compliance mechanism, the "brain" may also discover (dynamical) affordances of the physical world it is interacting with. In further experiments, the robot is connected to a revolvable bar or wheel with weights for giving it the some moment of inertia. In Video 8 the robotic arm finds a behavior rotating the wheel from an initial push by the user. When positioning the wheel in parallel to the arm, the modes were emerging even more readily as seen by Video9. Moreover, the system can immediately be switched between the forward and backward rotation mode. This is possible because the time-scale of the synaptic plasticity is so fast, in the order of one second, that the new dynamics is quickly propagating into the controller via the plasticity rule. By changing a time-scale meta-parameter the frequency of rotation can be adjusted, see Video 10 . The spontaneous emergence of the wheel rotation behavior can be argued to be a cognitive act if we consider-in the sense of (radical) embodied cognitive science - that cognition is to be described in terms of agent-environment dynamics and not in terms of computation and representation.

In another experimental situation, the robot is equipped with a brush and forced by manual guidance to wipe a table. Video 11 demonstrates how, by the combination of the limiting table plane and the manual force, the robot is driven into a two-dimensional wiping mode. This is seen to slowly wander through different wiping modes by the dynamics of the meta-system. Again, the manual interaction with the arm by little forces is always possible as seen later in the video. This is due to the tight closed loop control and the property of the synaptic dynamics to be compliant to external perturbances, see Der and Martius (2015). Most importantly, emerging motion patterns can be identified and stored by the user simply by taking snapshots of the synaptic weights. Video 12 shows the recall of previously acquired wiping modes. The transition between different modes is achieved by hard switching of the fixed controller weights, nevertheless smooth transients are observed.

In summary, we have treated a soft, high-complexity robotic system which a novel goal free exploratory control algorithm. It reverts the role of the controller and the controlled and makes a set of non-trivial and highly coordinated behaviors emerge solely from the interaction of synaptic dynamics, neural transmission and the mechatronic system. It provides a systematic approach for behavioral self-organization avoiding the reality gap as demonstrated by our applications to both simulated and real robots. In this way it can help to lift Artificial Life ecologies to a new level of complexity approaching physical reality of, say, humanrobot ecologies. The new controller may speed up evolution enormously Der and Martius (2015) as the emergence of a new trait needs only a mutation in morphology with adequate behaviors coming for free. Also the controller is fully deterministic revealing that behavioral proliferation can be the result of spontaneous symmetry breaking. Seen as a practical approach to generate complex, force-sensitive interactions with the environment this controller could also augment the repertoire of classical controllers. Additionally, it may shed light on how biological musculoskeletal systems generate the complex trajectories they use to interact with the environment with an unrivalled flexibility - not as a heavily controlled process but as an emergent phenomenon.

Supplementary material: playfulmachines.com/MyoArm-2 Acknowledgements: GM received funding from the EU (Marie Curie Actions, FP7, no. [291734]). RD gratefully acknowledges the hospitality in the group of Nihat Ay.

\section{References}

Der, R. and Martius, G. (2015). Novel plasticity rule can explain the development of sensorimotor intelligence. PNAS, 112(45):E6224-E6232. arXiv: abs/1505.00835.

Der, R. and Martius, G. (2016). The world as its own best controller: a case study with anthropomimetic robots. arXiv: abs/1602.02990. 